\title{
A Product Conceptual Evaluation Method Combining Interval Fuzzy TOPSIS and AHP
}

\author{
Lin Xiaohua, Jia Wenhua, Li Guo \\ School of Mechanical Engineering, Nanjing Institute of Technology, Nanjing, China \\ zdlxh5001@163.com
}

\begin{abstract}
Keywords: Conceptual evaluation; Interval fuzzy; Analytic hierarchy process; TOPSIS algorithm. Abstract. A product conceptual evaluation method combining analytic hierarchy process (AHP) and interval fuzzy technique for order preference by similarity to ideal solution (TOPSIS) was proposed in this paper. The weights of evaluation criteria were determined by AHP, where nine-level evaluation scales were defined to measure the importance of evaluation criteria and the judgment matrix was also constructed. We use interval fuzzy theory to express uncertain information for product performance evaluation, then we established the interval fuzzy evaluation matrix, based on which the improved ideal solution and the negative ideal solution was defined. On the basis of the above, the distances between each alternative to the positive and negative ideal solution were calculated, which were used to compute the clossness degree of each alternative to compare and rank the alternatives. At last, an illustrative example was discussed to validate the practicability and feasibility of the established evaluation method and theory.
\end{abstract}

\section{Introduction}

The product conceptual design results often deviate from customer expectations due to the diversity, fuzziness and uncertainty information of the customer demand in product conceptual design stage. So the conceptual product can not fully meet the customer needs, and there will be a number of alternative conceptual design. A scientific and reasonable conceptual product must satisfy the customer demand as far as possible, and reduce the possibility of faults in the life cycle of product manufacturing, maintenance and so on. Therefore, it is necessary to establish a set of scientific and effective evaluation theory and method in order to select the best scheme in most feasible alternatives.

At present, the research on the product conceptual evaluation mainly includes two aspects: the method of determining the weights of evaluation criteria and the method of decision making for conceptual design. Analytic hierarchy process, grey relational analysis and entropy method are the common research methods to determine criterion weight[1-3]. Among them, the AHP method has been widely used due to its convenience and flexibility, the key point of AHP is the judgment matrix, the elements of the matrix are the two-two contrast values of the relative importance of the evaluation criteria. So, AHP is a simple and feasible method to determine the weights as it combine qualitative evaluation with quantitative evaluation.

On the other hand, there are many researches on product decision making methods, mainly including TOPSIS, ELECTRE and all kinds of intelligent algorithm, etc[4-6]. TOPSIS proposed by C.L.Hwang and K.Yoon is a sorting method of approximation to the ideal solution[7]. Alternatives are sorted by the calculated distance between the evaluation object and the ideal solution and the negative ideal solution. The optimal solution is the most close to the ideal solution and the most far away from the negative ideal solution. TOPSIS has been widely used in the field of multi-attribute decision making as its advantages of simple calculation, reasonable evaluation results, flexible application and so on.

The selection of product conceptual scheme also belongs to multi-attribute decision making problem. Hence, this paper will combine interval fuzzy theory and TOPSIS methods to rank the alternative product schemes. It use AHP to calculate the weights of evaluation criteria, TOPSIS to construct multi-attribute rating matrix for product conceptual schemes. Where, the ideal solution and the negative ideal solution will be determined so as to calculate the closeness degree of alternative scheme 
to the ideal scheme. The alternatives are sorted according to the TOPSIS closeness degree, scheme with maximum closeness is the optimal one.

\section{Product conceptual evaluation model}

For the product conceptual evaluation, firstly, we should determine the performance evaluation values of each alternative as for certain evaluation criteria, then, the decision makers choose the best design scheme. Product conceptual evaluation model can be expressed in matrix F as follows:

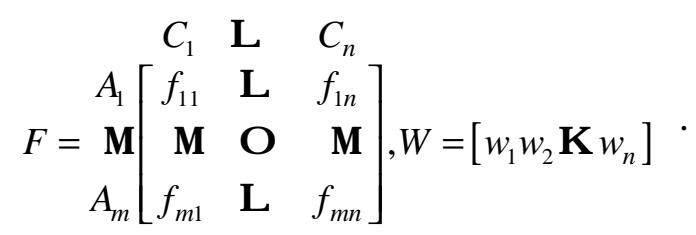

Where, $A_{1}, A_{2}, \ldots, A_{m}$ denote $m$ product alternative conceptual schemes for selection, $C_{1}, C_{2}, \ldots, C_{n}$ denote $n$ criteria in the product conceptual evaluation system, $f_{i j}$ is the evaluation value of scheme $A_{j}$ considering the criterion $C_{i}, w_{i}$ is the importance weight of criterion $C_{i}$. In this paper, AHP method and TOPSIS algorithm are combined to evaluate the product conceptual schemes, and the performance evaluation value is expressed in the form of interval fuzzy number. Where, the weights of evaluation criterion are determined by AHP method, and the conceptual scheme performance is evaluated by interval fuzzy TOPSIS.

\section{AHP method to determine the weights of evaluation criterion}

The relative importance between evaluation criterion is represented in the form of judgment matrix in AHP method, set $t_{i j}$ as the relative importance for criterion $i$ to criterion $j$, and it should satisfy the following condition: $t_{i j}>0, t_{i j}=1 / t_{j i}, t_{i i}=1$.

Therefore, the AHP judgment matrix is $\boldsymbol{T}=\left[t_{i j}\right]_{n \times n}$

For the judgment matrix $\boldsymbol{T}$, the root mean square method is usually used to calculate the weight vector:

$$
w_{i}=\sqrt[n]{\prod_{j=1}^{n} t_{i j}} / \sum_{i=1}^{n} \sqrt[n]{\prod_{j=1}^{n} t_{i j}}, i=1,2, \ldots, n
$$

Because the experts have a certain subjectivity in scoring, so the comparison between criterion is not possible to achieve the complete consistency, inevitably there will be some error. In order to prevent too large error, it is necessary to test the consistency of the judgment matrix, namely, we should calculate the random consistency ratio (CR) of the judgment matrix. When the consistency ratio is less than 0.1 , the judgment matrix can be determined to complete the consistency test.

\section{Interval fuzzy TOPSIS algorithm}

Technique for order preference by similarity to ideal solution (TOPSIS) is an effective method to solve multi-attribute decision problem. First, we should choose ideal solution and negative ideal solution from the alternative schemes. Then, the distance between each alternative scheme and positive ideal solution and the negative ideal solution are calculated, and so as the relative closeness degree computed to measure these two distance. Finally, alternative with maximum colseness degree is choosed as the optimal scheme. 
Now, there are $n$ alternative schemes $\left(z_{1}, z_{2}, \ldots, z_{n}\right)$ to be evaluated, m evaluation criteria $\left(c_{1}, c_{2}, \ldots\right.$, $\left.c_{m}\right)$, and $h$ experts $\left(D_{1}, D_{2}, \ldots, D_{h}\right)$ take part in evaluation. The specific steps are as follows:

1) construct the average decision matrix $\bar{Y}: \bar{Y}=\left[X_{i j}\right]_{n \times m}$.

$$
X_{i j}=\left(\left(x_{i j}^{1} \oplus x_{i j}^{2} \oplus \mathrm{L} \oplus x_{i j}^{h}\right) / h\right) .
$$

Where, $x_{i j}^{p}(p=1,2, \ldots, h)$ is the evaluation value of criterion $c_{j}$ for scheme $z_{i}$ by expert $\mathrm{D}_{\mathrm{p}}$, $X_{i j}=\left[x_{i j}^{L}, x_{i j}^{U}\right]$ is the average evaluation value, $1 \leq i \leq n, 1 \leq j \leq m$.

2) construct normalized decision matrix $\bar{Z}: \bar{Z}=\left[Z_{i j}\right]_{n \times m}$.

For benefit criterion, $c_{j} \in O$, the greater the evaluation value, the better the performance, and

$$
Z_{i j}=\left[\frac{x_{i j}^{L}-x_{i-}^{L}}{\text { length }_{j}}, \frac{x_{i j}^{U}-x_{i-}^{L}}{\text { length }}\right] .
$$

For cost criterion, $c_{j} \in I$, the smaller the evaluation value, the better the performance, and

$$
Z_{i j}=\left[\frac{x_{i+}^{U}-x_{i j}^{U}}{\text { length }_{j}}, \frac{x_{i+}^{U}-x_{i j}^{L}}{\text { length }_{j}}\right] \cdot
$$

Where, length $h_{j}=\max _{i} x_{i j}^{U}-\min _{i} x_{i j}^{L}, x_{i+}^{U}=\max _{i} x_{i j}^{U}, x_{i-}^{U}=\min _{i} x_{i j}^{L}$.

3) determine the ideal solution $\left(Z_{j}^{+}\right)$and the negative ideal solution $\left(Z_{j}^{-}\right)$for each evaluation criterion.

The ranking method of interval fuzzy numbers is used to sort the normalized evaluation values, according to which, the ideal solution and negative ideal solution of each criterion are as follows:

$$
\begin{aligned}
& Z_{j}^{+}=\left\{\begin{array}{ll}
\max _{i} Z_{i j} & c_{j} \in O \\
\min _{i} Z_{i j} & c_{j} \in I
\end{array} ;\right. \\
& Z_{j}^{-}=\left\{\begin{array}{ll}
\min _{i} Z_{i j} & c_{j} \in O \\
\max _{i} Z_{i j} & c_{j} \in I
\end{array} .\right.
\end{aligned}
$$

4) calculate the distance from the alternative to the positive and negative ideal solution. First , the distance from the normalized value $z_{i j}$ (evaluation value of criterion $c_{j}$ for scheme $z_{i}$ ) to the positive and negative ideal solution ( $Z_{j}^{+}$and $Z_{j}^{-}$respectively ) is computed as follows:

$$
d_{i j}^{+}=Z_{j}^{+} Z_{i j}, d_{i j}^{-}=Z_{i j}^{\ominus} Z_{j}^{-} .
$$

Then, the distance between the alternative schemes to the positive and negative ideal scheme is calculated respectively:

$$
D_{i}^{+}=w_{1} \otimes d_{i 1}^{+} \oplus w_{2} \otimes d_{i 2}^{+} \oplus \mathrm{L} \oplus w_{m} \otimes d_{i m}^{+} ; \quad D_{i}^{-}=w_{1} \otimes d_{i 1}^{-} \oplus w_{2} \otimes d_{i 2}^{-} \oplus \mathrm{L} \oplus w_{m} \otimes d_{i m}^{-} .
$$

5) calculate the relative closeness degree and its ranking value, and determine the optimal scheme. 


$$
\kappa_{i}=D_{i}^{-} d\left(D_{i}^{+} \oplus D_{i}^{-}\right)
$$

The value of $\kappa_{i}$ reflects the degree of deviation from the negative ideal scheme for the alternative scheme, the bigger the ranking value is, the greater the distance is, therefore, the alternative scheme with the maximum closeness degree is the best.

\section{Case study}

A company is developing a new type of rail vehicle door product. Now, there are 5 product conceptual schemes (denoted as $z_{1}, z_{2}, \ldots, z_{5}$ ) for selection, and 9 experts invited to take part in evaluation. 8 evaluation criteria are determined according to the market demand and design experience, namely, the performance of speed adjustment, resistance reducing, buffering, carriage sealing, noise reduction, braking, manufacturing cost and green initiative (denoted as $C_{1}, C_{2}, C_{3}, C_{4}$, $\mathrm{C}_{5}, \mathrm{C}_{6}, \mathrm{C}_{7}, \mathrm{C}_{8}$, respectively). Manufacturing cost is a cost criterion, the remaining 7 criteria are benefit criteria.

In the initial stage of the conceptual evaluation, the weights of evaluation criteria are obtained by AHP method, that is, 0.059, 0.199, 0.073, 0.216, 0.081, 0.062, 0.191, 0.119 (the weight calculation procedure is omitted). We uses 5-level interval fuzzy scale in the performance evaluation process, namely, $[0,0.2]$ for very low, $[0.2,0.4]$ for low, $[0.4,0.6]$ for medium, $[0.6,0.8]$ for high, $[0.8,1.0]$ for very high.

Tab.1 normalized decision matrix $\bar{Z}$

\begin{tabular}{|c|c|c|c|c|c|c|c|c|}
\hline & $\mathrm{C}_{1}$ & $\mathrm{C}_{2}$ & $\mathrm{C}_{3}$ & $\mathrm{C}_{4}$ & $\mathrm{C}_{5}$ & $\mathrm{C}_{6}$ & $\mathrm{C}_{7}$ & $\mathrm{C}_{8}$ \\
\hline$A$ & {$[0.302,0.345$} & $\begin{array}{l}{[0.295,0.337} \\
\end{array}$ & {$[0.315,0.363$} & {$[0.292,0.348$} & {$[0.232,0.274$} & {$[0.197,0.243$} & {$[0.261,0.313$} & {$[0.367,0.415$} \\
\hline$A_{1}$ & ] & ] & ] & ] & ] & ] & ] & ] \\
\hline $\mathrm{A}_{2}$ & {$[0.210,0.242$} & {$[0.237,0.284$} & {$[0.223,0.267$} & {$[0.315,0.353$} & {$[0.274,0.332$} & {$[0.205,0.257$} & {$[0.282,0.338$} & {$[0.127,0.165$} \\
\hline $\mathrm{A}_{2}$ & ] & ] & ] & ] & ] & ] & ] & ] \\
\hline $\mathrm{A}_{3}$ & {$[0.302,0.348$} & {$[0.273,0.325$} & {$[0.267,0.292$} & {$[0.297,0.342$} & {$[0.227,0.263$} & {$[0.308,0.384$} & {$[0.325,0.377$} & {$[0.298,0.332$} \\
\hline $\mathrm{A}_{4}$ & {$[0.260,0.307$} & {$[0.265,0.302$} & {$[0.260,0.315$} & {$[0.267,0.315$} & {$[0.317,0.357$} & {$[0.287,0.355$} & {$[0.253,0.307$} & $78,0.315$ \\
\hline $\mathrm{A}_{5}$ & {$[0.345,0.377$} & {$[0.262,0.312$} & {$[0.255,0.295$} & {$[0.347,0.384$} & {$[0.405,0.463$} & {$[0.238,0.289$} & {$[0.298,0.342$} & {$[0.252,0.295$} \\
\hline
\end{tabular}

The analysis steps of interval fuzzy TOPSIS method are as follows:

1) Experts assess the performance of the alternative schemes, the results are processed by formula (3) to get the average decision matrix, the normalized decision matrix is calculated per formula (4) and (5), as shown in Tab.1

2) According to formula (6) and (7), we can determine the ideal solution and the negative ideal solution of each evaluation criterion as follows: $Z_{1}^{+}=z_{51}, Z_{2}^{+}=z_{12}, Z_{3}^{+}=z_{13}, Z_{4}^{+}=z_{54}, Z_{5}^{+}=z_{55}, Z_{6}^{+}=z_{36}, Z_{7}^{+}=z_{37}, Z_{8}^{+}=z_{18}$; $Z_{1}^{-}=z_{21}, Z_{2}^{-}=z_{22}, Z_{3}^{-}=z_{23}, Z_{4}^{-}=z_{44}, Z_{5}^{-}=z_{35}, Z_{6}^{-}=z_{16}, Z_{7}^{-}=z_{47}, Z_{8}^{-}=z_{28}$.

3) Calculate distance of each alternative to positive and negative ideal solution per formula (8) and (9), based on these distances, we compute the closeness degree per formula (10), results are as follows: $\kappa_{1}=\left[\begin{array}{ll}0.069 & 0.535\end{array}\right], \kappa_{2}=\left[\begin{array}{lll}-0.125 & 0.580\end{array}\right], \kappa_{3}=\left[\begin{array}{lll}0.090 & 0.545\end{array}\right], \kappa_{4}=\left[\begin{array}{ll}-0.015 & 0.443\end{array}\right], \kappa_{5}=[0.1410 .540]$. We apply the fuzzy number comparison method to sort the alternative schemes, the descending order is $A_{4} 、 A_{1} 、 A_{3} 、 A_{5}$ 、 $\mathrm{A}_{2}$.

4) In order to verify effectivity of the interval fuzzy TOPSIS method, interval fuzzy VIKOR method, interval fuzzy COPRAS method and traditional TOPSIS method are employed for this case study, the ranking results are shown in table 2. Calculate the Spearman rank correlation coefficients of the ranking by proposed method with ranking by interval fuzzy VIKOR、 interval fuzzy COPRAS, that is, 1.00 and 0.90 respectively, as for traditional TOPSIS, the two coefficients are 0.90 and 0.80 , respectively. Therefore, compared with the traditional TOPSIS method, the interval fuzzy TOPSIS 
method is more similar to the ranking results of the interval fuzzy VIKOR method and the interval fuzzy COPRAS method.

Tab. 2 scheme ranking results of the four methods

\begin{tabular}{llllll}
\hline Alternative schemes & $\mathrm{Z}_{1}$ & $\mathrm{Z}_{2}$ & $\mathrm{Z}_{3}$ & $\mathrm{Z}_{4}$ & $\mathrm{Z}_{5}$ \\
\hline Interval fuzzy TOPSIS & 2 & 5 & 3 & 1 & 4 \\
Interval fuzzy VIKOR & 2 & 5 & 3 & 1 & 4 \\
Interval fuzzy COPRAS & 2 & 5 & 4 & 1 & 3 \\
Traditional TOPSIS & 3 & 4 & 2 & 1 & 5 \\
\hline
\end{tabular}

\section{Conclusions}

1) The product conceptual evaluation is a multi-attribute decision problem, which needs to consider the multiple evaluation criteria of the product.

2) As for the expression deficiency of uncertain information by traditional theory, this paper proposed a product conceptual evaluation method based on interval fuzzy TOPSIS and AHP method.

3) The application example shows that the product conceptual evaluation method based on AHP and interval fuzzy TOPSIS is feasible and effective.

\section{Acknowledgements}

This work was financially supported by the National Natural Science Foundation of China (No.51405222) and Imported Talent Scientific Research Foundation of Nanjing Institute of Technology (No.YKJ201301).

\section{References}

[1] Ezzat A E M, Hamoud H S. French Review, 2016, 6(1):3-7.

[2] Qin J, Zhang Y. Pharmacogenetics \& Genomics, 2015, 15(11):769-78.

[3] Ayağ Z, Özdemir R G. Computers \& Industrial Engineering, 2009, 56(1): 368-379.

[4] Lin M C, Wang C C, Chen M S, et al. Computers in Industry, 2008, 59(1): 17-31.

[5] Chen T Y, Tsao C Y. Fuzzy Sets and Systems, 2008, 159(11): 1410-1428.

[6] Malekmohammadi B, Zahraie B, Kerachian R. Expert Systems with Applications, 2011, 38(6): 7851-7863.

[7] Huang C L,Yoon K. Multi-attribute decision making: methods and applications. NY: springer-Verlag, 1981: 12-34. 